\title{
RESPUESTA AGRONÓMICA DE Zea mays L. y Phaseolus vulgaris L. A LA FERTILIZACIÓN CON COMPOST
}

\author{
NATALIA ESCOBAR ESCOBAR ${ }^{1}$ \\ JAIRO MORA DELGADO ${ }^{2}$ \\ NÉSTOR ROMERO JOLA ${ }^{3}$
}

Recibido el 24 de septiembre de 2012 y aprobado el 4 de febrero de 2013

\begin{abstract}
RESUMEN
El compostaje es una tecnología ecológica, la cual constituye una vía para la reutilización y biotransformación de materiales orgánicos así como su posterior aplicación en suelos. La calidad de un abono orgánico se determina a partir del contenido nutricional y de la capacidad de proveer nutrientes a un cultivo. Con base en un compostaje de material orgánico en mezcla (pulpa de café, residuos de banano, gallinaza y bovinaza), proveniente de fincas cafeteras ubicadas en el departamento de Cundinamarca, se determinaron y compararon indicadores químicos y biológicos. Además, mediante un bioensayo, usando dos especies con alto potencial alimentario y forrajero: maíz (Zea mays L.) y fríjol (Phaseolus vulgaris L.), se evaluó el efecto del compost sobre parámetros agronómicos y productividad en ambos cultivos. Siete tratamientos fueron evaluados [Abono químico (Q), Mezcla 1 (Mz1), Mezcla 2 (Mz2), Mezcla 3 (Mz3), Testigo sin fertilización (SF), Abono orgánico comercial (AOC)], con base en la determinación de parámetros químicos ( $\mathrm{MO}$, macro y micronutrientes). En el bioensayo con maíz (Zea mays L.), Q y Mz1 obtuvieron los parámetros más altos durante la etapa vegetativa; en la etapa de floración la Mz3 obtuvo los mejores valores en los parámetros. En fríjol (Phaseolus vulgaris L.), en la etapa vegetativa, los tratamientos Q y Mz1 fueron los mejores sin presentar diferencias significativas entre sí; en las etapas de floración y rendimiento Mz2 fue la que obtuvo mejor respuesta. En conclusión, los abonos orgánicos son una alternativa viable y sostenible probada bajo condiciones de campo.
\end{abstract}

\section{PALABRAS CLAVE}

Compostaje, gramínea, leguminosa, parámetros agronómicos, productividad.

AGRONOMIC RESPONSE OF Zea mays L. and Phaseolus vulgaris L. TO COMPOST FERTILIZATION

\section{ABSTRACT}

Composting is an ecological technology which constitutes a way to reusing and biotransforming organic materials as well as their subsequent application in soil. The quality of organic fertilizer is determined from the nutritional content and the ability to provide nutrients to a crop. Based on an organic material composting mix, (coffee pulp, banana waste, chicken and cattle manure) coming from the coffee farms located in the department of Cundinamarca, chemical and biological indicators were determined and compared. In addition, through a bioassay, using two species with high food and feed potential, corn (Zea mays L.) and bean (Phaseolus vulgaris L.), the effect of compost on agronomic parameters and productivity in both crops 
was assessed. Seven treatments were evaluated [chemical fertilizer (Q), mixture 1 (Mz1), mixture 2 (Mz2), mixture 3 (Mz3), control without fertilizer (SF), commercial organic fertilizer (AOC)], based on the determination of chemical parameters ( $\mathrm{MO}, \mathrm{CO}$, macro and micronutrients). In the bioassay with corn (Zea mays L.), Q and Mz1 obtained the highest parameters during the vegetative stage; in the flowering stage, Mz3 obtained the best values in the parameters. In beans (Phaseolus vulgaris L.) in the vegeteative stage treatments, Q and Mz1 were the best, without showing statistical differences among them. In the flowering and harvesting phase, Mz2 obtained a better response. In conclusion, organic fertilizers are a viable and sustainable alternative tested under field conditions.

\section{KEY WORDS}

Composting, grasses, legumes, agronomic parameters, productivity.

\section{INTRODUCCIÓN}

Los abonos orgánicos constituyen una forma de reciclaje de nutrientes en el sistema agropecuario, estos incluyen todo material de origen orgánico utilizado para la fertilización de cultivos o como mejoradores de suelos (Soto, 2003). El compostaje es una tecnología ecológica que permite la reutilización y biotransformación de materiales orgánicos; el compost en la etapa final del proceso adquiere su madurez cuando se obtiene un producto estable (Labrador, 2001). Un compost es un recurso orgánico capaz de proporcionar cantidades notables de nutrientes esenciales, principalmente nitrógeno, fósforo y potasio al suelo o a las plantas (Gómez, 2000), estos mejoran las propiedades físicas, químicas y biológicas del suelo. Los efectos del compost sobre las propiedades físicas van dirigidos hacia tres objetivos concretos: el mejoramiento de la estabilidad estructural, la regulación del balance hídrico del suelo y el mejoramiento de las propiedades químicas. En este último aspecto, los abonos orgánicos aumentan el poder tampón del suelo reduciendo las oscilaciones de $\mathrm{pH}$; de otro lado, incrementan la capacidad de intercambio catiónico mejorando la fertilidad.

Con relación a las propiedades biológicas, los abonos orgánicos favorecen la aireación y oxigenación del suelo, promoviendo una mayor actividad radicular y facilitando la dinámica de microorganismos aerobios. De esta manera, constituyen una fuente de energía para que los microorganismos se multipliquen rápidamente (Kolmans \& Vásquez, 1996). La calidad del compost está afectada por la calidad del material original, como grado de digestión, contenido original de nutrientes, entre otros, y por el sistema de compostaje utilizado (Mazzarino et al., 2005). Para evaluar la calidad de los materiales orgánicos, durante y al final del proceso de compostaje, se han propuesto diferentes criterios basados en la cuantificación de los parámetros físicos, químicos y biológicos (Cegarra, 1994). Estos criterios definen las características benéficas del compost y permiten recomendar su aplicación para diferentes finalidades agrícolas, siendo esta última la forma de evaluar los efectos del producto sobre variables de rendimiento.

Las prácticas agronómicas de fertilización hacen referencia a todas aquellas técnicas que permiten mejorar la fertilidad de las tierras desde el punto de vista físico, químico y biológico (Bertsch, 2003). Dentro de estas, 
el abastecimiento de nutrimentos se realiza a través de fuentes minerales (fertilizantes sintéticos) y abonos orgánicos como los estiércoles, restos de cosecha, compost y vermicompost, entre otros. En las últimas décadas ha cobrado importancia el uso de fuentes orgánicas debido a los costos de los fertilizantes químicos, al desequilibrio ambiental que estos ocasionan en los suelos y a la necesidad de preservar la materia orgánica en los sistemas agrícolas, lo cual es un aspecto fundamental relacionado con la sostenibilidad de dichos sistemas (Ramírez, 2005).

El objetivo de este estudio fue comparar el efecto del compost obtenido a partir de tres mezclas de residuos orgánicos provenientes de fincas cafeteras, en indicadores agronómicos de las especies maíz (Zea mays L.) y fríjol (Phaseolus vulgaris L.).

\section{MATERIALES Y MÉTODOS}

El proyecto se llevó a cabo en un invernadero de $1500 \mathrm{~m}^{2}$, ubicado en el municipio de Fusagasugá (Cundinamarca). Allí se evaluaron comparativamente las mezclas, frente a un abono orgánico comercial, uno químico y un control (solo suelo). La siembra de las especies Zea mays L. y Phaseolus vulgaris L. se realizó a una distancia entre planta de $80 \mathrm{~cm}$ y entre surco un metro. La dosis de compostaje aplicado al suelo se calculó a partir del resultado obtenido en el análisis de suelo y los requerimientos de las dos especies. Se evaluaron las variables de respuesta dos veces por semana comparadas con tres testigos (Triple 15, FQ; abono orgánico comercial, AOC; y sin fertilizante, SF), aplicadas en forma de corona en el momento de siembra y en etapa de prefloración (en las etapas fenológicas descritas en la Tabla 1).

Tabla 1. Etapas fenológicas a evaluar

\begin{tabular}{cccc}
\hline CULTIVOS & \multicolumn{3}{c}{ ETAPA FENOLÓGICA } \\
\hline MAiz (Zea mays L.) & $\begin{array}{c}\text { V0: Germinación y } \\
\text { emergencia }\end{array}$ & V4: Prefloración & V6: Antesis \\
FRiJOL (Phaseolus vulgaris L.) & $\begin{array}{c}\text { V0: Germinación y } \\
\text { emergencia }\end{array}$ & V3: Prefloración & V4: Antesis \\
\hline
\end{tabular}

Se eligieron específicamente estas etapas por la importancia del suministro de abonos en la fase de inicio de cada cultivo y por sus estados de máxima translocación de asimilados. Para determinar los efectos de las mezclas aplicadas sobre las etapas fenológicas de los dos cultivos se midieron las variables descritas en la Tabla 2. 
Tabla 2. Variables a medir según etapa fenológica

\begin{tabular}{|c|c|c|}
\hline ETAPA FENOLOGICA & CULTIVO & VARIABLE \\
\hline V0 (Germinación) & Maíz-Fríjol & $\begin{array}{l}* \begin{array}{l}\text { Porcentaje de germinación, tasa } \\
\text { de germinación, vigor. }\end{array}\end{array}$ \\
\hline V3 (Prefloración) & Frijol & $\begin{array}{l}\text { * Tasas fisiológicas: TCR (Tasa de } \\
\text { Crecimiento Relativo), AFE (Area } \\
\text { Foliar Especifica). }\end{array}$ \\
\hline V4 (Prefloración) & Maíz & $\begin{array}{l}\text { * Variables morfológicas: Peso } \\
\text { seco, peso fresco del total de la } \\
\text { planta. }\end{array}$ \\
\hline V4 (Antesis) & Frijol & $\begin{array}{l}\text { * Tasas fisiológicas: TCR (Tasa de } \\
\text { Crecimiento Relativo), AFE (Area } \\
\text { Foliar Especifica). }\end{array}$ \\
\hline V6 (Antesis) & Maíz & $\begin{array}{l}\text { * Variables morfológicas: Peso } \\
\text { seco, peso fresco del total de la } \\
\text { planta. }\end{array}$ \\
\hline \multirow[t]{2}{*}{ Finalización ciclo del cultivo } & Frijol & $\begin{array}{l}* \text { Rendimiento: Número y peso de } \\
\text { vainas por planta. }\end{array}$ \\
\hline & Maíz & $\begin{array}{l}* \text { Rendimiento: Número y peso de } \\
\text { mazorcas por planta. }\end{array}$ \\
\hline
\end{tabular}

Para medir las variables se utilizaron las siguientes ecuaciones (Hunt, 2002):

Para Peso Seco (PS) se utilizó la siguiente fórmula:

PS $=$ peso fresco - peso final

Para Área Foliar (AF) se aplicó la fórmula de Simpson donde

$\mathrm{AF}=\frac{\text { área de la hoja }}{2}$

Para İndice de Área Foliar (IAF) se utilizó la siguiente fórmula:

$I A F=\frac{\text { área foliar }}{\text { área del suelo }}$

Para Tasa de Crecimiento Relativo (TCR) se aplicó la fórmula:

$\mathrm{TCR}=\frac{\operatorname{LogN} \mathrm{Peso}_{2}-\operatorname{LogN~peso~}_{1}}{\text { tiempo }_{2}-\text { tiempo }_{1}}$

Para Tasa de Crecimiento del cultivo (TCC) se utilizó la fórmula:

$\mathrm{TCC}=\frac{\mathrm{PS}_{1} \times \text { peso }_{2}-\text { peso }_{1}}{\text { tiempo }_{2}-\text { tiempo }_{1}}$

Para Tasa de Asimilación Neta (TAN) se aplicó la fórmula

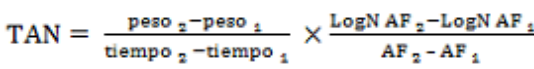

Para Área Foliar Específica (AFE) se utilizó la fórmula

$\mathrm{AFE}=\frac{\text { área foliar }}{\text { peso seco }}$ 
El riego se realizó de acuerdo con las necesidades hídricas de las plantas en las etapas de germinación, floración y fructificación.

Se evaluaron tres mezclas de sustratos orgánicos balanceadas, para obtener una relación $\mathrm{C} / \mathrm{N}$ óptima para compostaje (Tabla 3), con base en los residuos de mayor disponibilidad en las fincas cafeteras: banano de rechazo, bovinaza, pulpa de café gallinaza. Las mezclas se estandarizaron a $85 \mathrm{~kg}$ y se adicionaron a cada tratamiento $10 \mathrm{~g}$ de melaza diluida para estimular la actividad microbiana y $10 \mathrm{~g}$ de inóculo (compost terminado de gallinaza) por cada $100 \mathrm{~g}$ de muestra para promover la actividad microbiana y reducir el tiempo de maduración (Jia et al., 2011).

Tabla 3. Proporción de materiales orgánicos y relación $\mathrm{C} / \mathrm{N}$ en tres mezclas compostadas

\begin{tabular}{|c|c|c|c|c|c|c|c|}
\hline \multirow[b]{2}{*}{ Mezcla } & \multirow[b]{2}{*}{ Medida (kg) } & \multicolumn{5}{|c|}{ Ingredientes } & \multirow{2}{*}{$\mathrm{C} / \mathrm{N}$} \\
\hline & & Pulpa café & Bovinaza & Gallinaza & Banano & Hojarasca & \\
\hline \multirow{2}{*}{1} & Peso fresco & 15,0 & & 15,0 & 5,0 & 50,0 & \multirow{3}{*}{27} \\
\hline & Materia seca & 6,0 & & 12,8 & 1,0 & 40,0 & \\
\hline \multirow{2}{*}{2} & Peso fresco & 15,0 & 15,0 & & 5,0 & 50,0 & \\
\hline & Materia seca & 6,0 & 12,8 & & 1,0 & 40,0 & \multirow[t]{2}{*}{27} \\
\hline \multirow{2}{*}{3} & Peso fresco & 8,5 & 17,0 & 17,0 & & 42,5 & \\
\hline & Materia seca & 4.0 & 15.0 & 15.0 & & 34.0 & 27 \\
\hline
\end{tabular}

Mezcla 1 = Pulpa + Hojarasca + Banano + Gallinaza; Mezcla 2 = Pulpa + Hojarasca + Banano + Bovinaza; Mezcla 3 = Pulpa + Hojarasca + Gallinaza + Bovinaza.

\section{ANÁLISIS ESTADÍSTICO}

Para evaluar el efecto de las mezclas finales se utilizó un modelo de bloques aleatorios con arreglo de subparcelas, en el que se definió la parcela como especie (Zea mays L. y Phaseolus vulgaris L.) y las subparcelas como los tratamientos ( $M z 1, M z 2, M z 3, F Q$ y AOC). Con el propósito de reducir el error experimental se implementaron tres repeticiones por tratamiento. Para evaluar los tratamientos se aplicó el procedimiento GLM del paquete estadístico SAS versión 9.0. Las variables dependientes en la evaluación fueron: porcentaje de germinación, tasa de crecimiento relativo, área foliar específica, peso seco, peso fresco y rendimiento en número y peso de frutos. Cuando se registró efecto significativo (alfa del 5\%) de los factores experimentales, se procedió a realizar pruebas de comparación de medias de Tukey, por medio del mismo programa estadístico.

\section{RESULTADOS Y DISCUSIÓN}

\section{Parámetros químicos del compost}

El mayor contenido de nutrientes mostrado en las mezclas 1 y 3 es consecuente con los mayores contenidos de materia orgánica en las mismas (Tabla 4). Los elementos N, P y K presentaron valores dentro del rango usual en los tres tratamientos, pero se detectaron diferencias significativas $(p<0,05)$ entre los distintos tratamientos para $N$ y $P$, mientras que $\mathrm{K}$ no presentó diferencias. Pierre et al. (2009) obtuvieron valores de $\mathrm{N}$ $(4,4-5,9 \%)$ y K $(2,5-3,6 \%)$ superiores a los hallados en esta investigación, posiblemente porque las mezclas de ese estudio contenían altas 
proporciones de estiércol, sin mezcla de sustratos vegetales fibrosos, lo cual puede ejercer como factor de dilución.

La riqueza de macronutrientes en el compost puede atribuirse a la presencia de gallinaza y pulpa de café, de acuerdo con lo reportado por Liang, Das y McClendon (2003), quienes sostienen que estos materiales son ricos en macro y micronutrientes, principalmente la gallinaza resultante de la de cría de gallinas ponedoras en jaula, que se considera un sustrato de alta calidad nutricional (Raviv et al., 2004), como la utilizada en la presente investigación. En el caso de la pulpa de café, Julca-Otiniano, Solano-Arrue y Crespo-Costa (2008) reportan altos valores de $\mathrm{P}(0,28 \%), \mathrm{K}$ $(2,5 \%)$ y MO (91\%), esta última atacada por los microorganismos del suelo que la biodegradan y constituyen un importante reservorio lábil de $\mathrm{C}, \mathrm{N}$ y $\mathrm{P}$ (Díaz, Acea \& Carballas, 1993).

Tabla 4. Resultados de parámetros químicos para mezclas

\begin{tabular}{lcccc}
\hline \multicolumn{1}{c}{ PARAMETROS } & UNIDAD & $\mathrm{Mz} 1$ & $\mathrm{Mz2}$ & $\mathrm{Mz} 3$ \\
\hline Materia Orgánica & $\%$ & $38,65 \mathrm{a}$ & $34,87 \mathrm{~b}$ & $37,76 \mathrm{a}$ \\
Nitrógeno orgánico & $\%$ & $1,88 \mathrm{a}$ & $1,64 \mathrm{~b}$ & $1,84 \mathrm{a}$ \\
Relación C/N & $\%$ & $11,5 \mathrm{a}$ & $10 \mathrm{~b}$ & $11,1 \mathrm{a}$ \\
C.I.C. & $\mathrm{meq} / 100 \mathrm{~g}$ & $27,24 \mathrm{~b}$ & $27,44 \mathrm{~b}$ & $30,58 \mathrm{a}$ \\
Fósforo & $\% \mathrm{P}$ & $4,84 \mathrm{c}$ & $8,04 \mathrm{a}$ & $7,18 \mathrm{~b}$ \\
Potasio & $\% \mathrm{~K}$ & $1,91 \mathrm{a}$ & $1,99 \mathrm{a}$ & $1,99 \mathrm{a}$ \\
Calcio & $\% \mathrm{Ca}$ & $1,19 \mathrm{a}$ & $0,47 \mathrm{~b}$ & $0,379 \mathrm{c}$ \\
Magnesio & $\% \mathrm{Mg}$ & $0,045 \mathrm{a}$ & $0,179 \mathrm{a}$ & $0,145 \mathrm{a}$ \\
Azufre & $\% \mathrm{~S}$ & $0,368 \mathrm{~b}$ & $0,506 \mathrm{a}$ & $0,39 \mathrm{~b}$ \\
Hierro & $\mathrm{mg} \mathrm{Fe} / \mathrm{kg}$ & $198 \mathrm{a}$ & $70,27 \mathrm{c}$ & $141 \mathrm{~b}$ \\
Manganeso & $\mathrm{mg} \mathrm{Mn} / \mathrm{kg}$ & $288 \mathrm{a}$ & $202 \mathrm{~b}$ & $310 \mathrm{a}$ \\
Cobre & $\mathrm{mg} \mathrm{Cu} / \mathrm{kg}$ & $0,895 \mathrm{c}$ & $1,39 \mathrm{~b}$ & $1,76 \mathrm{a}$ \\
\hline \multicolumn{1}{c}{ Fuente: Escobar (2011). } & & & &
\end{tabular}

Una adecuada relación $\mathrm{C} / \mathrm{N}$, favorecerá un buen crecimiento y reproducción microbiana, así Pérez et al. (2006) mencionan que la relación C/N óptima para el inicio del compostaje en mezclas está comprendida entre 25-35/1, esta relación se reduce a valores cercanos a 10-15/1, cuando el material está listo para ser usado, valores similares a los manejados en el presente estudio (10-12) para las tres mezclas, lo cual es indicador del estado de estabilidad de la materia orgánica como lo reportan Illner et al. (2007), sugiriendo que están listos para ser utilizados como abono.

\section{Respuesta agronómica de Maíz (Zea mays L.)}

La Tabla 5 describe comparativamente los valores obtenidos en cada tratamiento con respecto a las variables de crecimiento obtenidas en el cultivo de maíz. 
Tabla 5. Variables de crecimiento etapa vegetativa de maíz

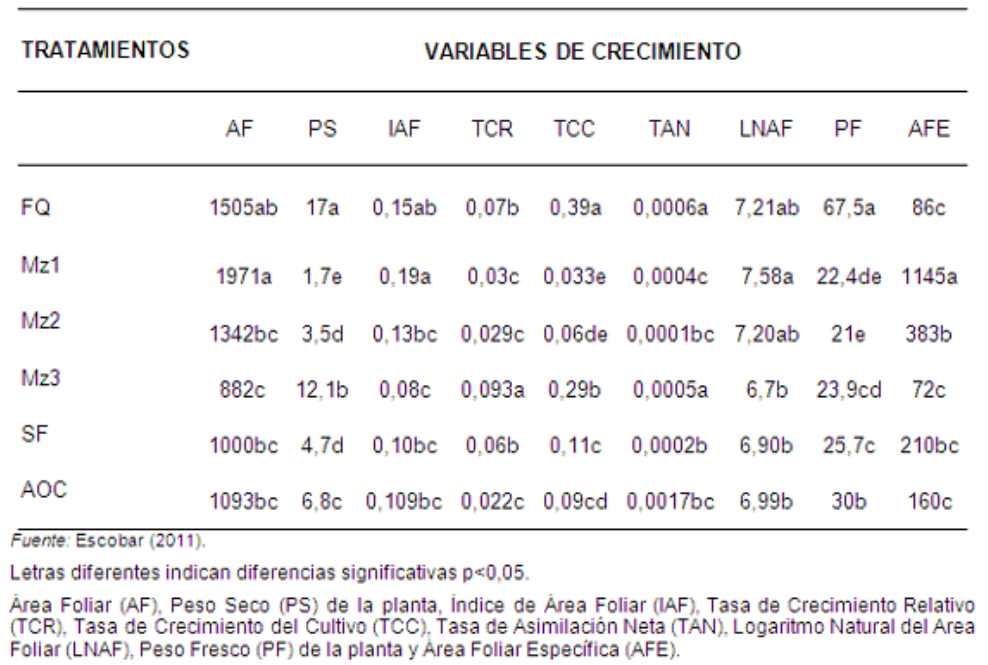

En general, los mejores resultados de las variables de crecimiento en la etapa vegetativa se presentaron en el tratamiento químico y mezcla 1, lo que indica que el abono orgánico, representado en la mezcla, tuvo un efecto casi similar al químico. Esto sugiere una alternativa de fertilización en el cultivo de maíz. Zamora y Benavides (2002), realizaron una evaluación del efecto de la fertilización mineral y orgánica (gallinaza) en el crecimiento y rendimiento del cultivo de maíz, logrando los mejores rendimientos en el tratamiento con gallinaza, similar a esta investigación donde la mezcla 1 contenía gallinaza. Al respecto García y Monje (1995) mencionan que los nutrientes contenidos en la gallinaza se tornan más aprovechables y disponibles para la planta en la etapa de crecimiento, ya que esta presenta mayor área radicular y por ende mayor acceso a la absorción de los elementos nutritivos liberados por el estiércol.

Adicionalmente, la mezcla 3 tuvo el segundo valor más alto en contenido de materia orgánica $(37,76 \%)$, valor que puede estar relacionado con el efecto de esta sobre las plantas de maíz, ya que esta fuente nutricional no solo debe verse como una fuente portadora de elementos nutritivos asimilables para las plantas, sino como compuesto de una acción activa que incide directamente en el mejoramiento de los suelos, mejorando su estructura, estabilidad, aireación, permeabilidad, retención de agua aprovechable, actividad microbiana, cediendo lentamente el nitrógeno y formando compuestos que facilitan la asimilación de elementos como el fósforo y su preservación (Butler, McFarland \& Muir, 2000). En la fase de floración (Tabla 6) la mezcla 3 fue la que mejor respuesta tuvo en las variables AF, PS, TCR, TCC, TAN, LNAF, PF y AFE presentando diferencias significativas $(p<0,05)$. 
Tabla 6. Variables de crecimiento etapa de floración en maíz

\begin{tabular}{|c|c|c|c|c|c|c|c|c|c|}
\hline \multirow[t]{2}{*}{ TRATAMIENTOS } & \multicolumn{9}{|c|}{ VARIABLES DE CRECIMIENTO } \\
\hline & AF & PS & IAF & TCR & $\mathrm{TCC}$ & TAN & LNAF & PF & AFE \\
\hline $\mathrm{FQ}$ & 1701ab & $19 a$ & $0,17 a b$ & $0,09 \mathrm{~b}$ & $0,40 \mathrm{~b}$ & $0,0008 \mathrm{a}$ & $8,31 \mathrm{ab}$ & $80,3 \mathrm{~b}$ & $1020 \mathrm{~b}$ \\
\hline Mz1 & $2081 \mathrm{a}$ & $9,6 b$ & $0,21 \mathrm{a}$ & $0,098 \mathrm{a}$ & $0,42 \mathrm{a}$ & $0,0005 b$ & $8,68 \mathrm{a}$ & $78,4 b$ & $1122 b$ \\
\hline Mz2 & $1451 b c$ & $4,5 c$ & $0,15 b c$ & $0,042 \mathrm{bc}$ & $0,36 b$ & $0,0003 b c$ & $8,20 \mathrm{ab}$ & $48 \mathrm{c}$ & $952 b c$ \\
\hline $\mathrm{Mz3}$ & $1113 a$ & $18,2 \mathrm{a}$ & $0,16 b$ & $0,010 \mathrm{a}$ & $0,43 a$ & $0,0007 a$ & $7,97 \mathrm{a}$ & $85,4 a$ & $1241 \mathrm{a}$ \\
\hline SF & $1090 \mathrm{c}$ & $6,2 \mathrm{bc}$ & $0,11 \mathrm{bc}$ & $0,08 \mathrm{~b}$ & $0,19 \mathrm{c}$ & $0,0002 b$ & $7,12 \mathrm{~b}$ & $35,9 \mathrm{c}$ & $210 \mathrm{c}$ \\
\hline $\mathrm{AOC}$ & $1085 b c$ & $7,6 c$ & $0,119 b c$ & $0,031 \mathrm{c}$ & $0,38 \mathrm{~b}$ & $0,0004 b c$ & $7,56 \mathrm{~b}$ & $68 b c$ & $960 \mathrm{bc}$ \\
\hline \multicolumn{10}{|c|}{ Fuente: Escobar (2011). } \\
\hline \multicolumn{10}{|c|}{ Letras diferentes indican diferencias significativas $p<0,05$. } \\
\hline $\begin{array}{l}\text { Area Foliar (AF), Pe } \\
\text { (TCR), Tasa de Cred } \\
\text { Foliar (LNAF), Peso }\end{array}$ & $\begin{array}{l}\text { co (PS } \\
\text { to del } \\
\text { (PF) d }\end{array}$ & 6) de la & planta, İno & tim & clíca & AF) & e $\mathrm{Cr}$ & ent & elativo \\
\hline
\end{tabular}

La Tabla 7 muestra comparativamente el número promedio de mazorcas por planta y peso promedio de mazorcas por planta para cada tratamiento. El número no presenta diferencias significativas $(p<0,005)$ entre tratamientos. Con respecto al peso la mezcla 3 obtuvo el mayor valor y presentó diferencias significativas $(p<0,005)$ con respecto al resto de tratamientos. El segundo valor lo obtuvo el testigo y el abono orgánico comercial; el tratamiento químico y las mezclas 1 y 2 no presentaron diferencias significativas $(p<0,005)$.

Tabla 7. Variables de rendimiento del producto final en maíz

\begin{tabular}{ccc}
\hline TRATAMIENTOS & No. de mazorcas & Peso mazorca \\
\hline FQ & $1,5 \mathrm{a}$ & $397,5 \mathrm{ab}$ \\
Mz1 & $1,5 \mathrm{a}$ & $346 \mathrm{ab}$ \\
Mz2 & $2,0 \mathrm{a}$ & $502,7 \mathrm{ab}$ \\
Mz3 & $2,5 \mathrm{a}$ & $611,2 \mathrm{a}$ \\
SF & $1,25 \mathrm{a}$ & $303,2 \mathrm{~b}$ \\
AOC & $2,1 \mathrm{a}$ & $322,2 \mathrm{~b}$ \\
\hline
\end{tabular}

Letras diferentes indican diferencias significativas $p<0,05$.

El tratamiento que generó mayor rendimiento fue la mezcla 3, esto posiblemente se deba a su composición basada en estiércoles (bovinaza y gallinaza), los cuales son abonos orgánicos concentrados y de rápida acción, ya que sus nutrientes se encuentran en compuestos asimilables por la planta (Eghball, Ginting \& Gilley, 2004); el periodo transcurrido desde la aplicación hasta la floración del cultivo, es tiempo suficiente para que se produzca una mineralización y por consiguiente un aporte de nutrimentos que puede coincidir con el periodo de mayor demanda de nutrientes.

\section{Respuesta agronómica de fríjol (Phaseolus vulgaris L.)}

La Tabla 8 describe comparativamente los valores obtenidos en cada tratamiento con respecto a las variables de crecimiento AF, PS, IAF, TCR, TCC, TAN, LNAF, PF y AFE obtenidas en el cultivo de fríjol. 
Tabla 8. Variables de crecimiento etapa vegetativa de frijol

\begin{tabular}{|c|c|c|c|c|c|c|c|c|c|}
\hline \multirow[t]{2}{*}{ TRATAMIENTOS } & \multicolumn{9}{|c|}{ VARIABLES DE CRECIMIENTO } \\
\hline & $\mathrm{AF}$ & PS & IAF & TCR & $\mathrm{TCC}$ & TAN & LNAF & PF & AFE \\
\hline FQ & $124,61 \mathrm{a}$ & $26,42 a$ & $0,012 \mathrm{a}$ & $0,05 b$ & $0,58 \mathrm{a}$ & $0,013 \mathrm{a}$ & $4,82 a$ & $54,77 \mathrm{a}$ & $4,71 \mathrm{c}$ \\
\hline Mz1 & $132,9 a$ & $24,26 b$ & $0,012 \mathrm{a}$ & $0,07 a$ & $0,57 a$ & $0,012 \mathrm{a}$ & $4,81 \mathrm{a}$ & $53,29 a$ & $5,10 \mathrm{c}$ \\
\hline $\mathrm{Mz2}$ & $71,47 \mathrm{c}$ & $6,73 \mathrm{c}$ & $0,007 \mathrm{c}$ & $0,046 \mathrm{bc}$ & $0,142 \mathrm{~b}$ & $0,005 c$ & $4,26 \mathrm{c}$ & $40,79 b$ & $10,72 a$ \\
\hline $\mathrm{Mz} 3$ & $85,45 b$ & $7,03 \mathrm{c}$ & $0,008 \mathrm{~b}$ & $0,041 \mathrm{c}$ & $0,142 \mathrm{~b}$ & $0,004 \mathrm{c}$ & $4,44 b$ & $38,99 b$ & $12,14 a$ \\
\hline SF & $50,4 d$ & $4,75 d$ & $0,005 d$ & $0,044 b c$ & $0,09 \mathrm{c}$ & $0,004 c$ & $3,91 \mathrm{~d}$ & $27,52 \mathrm{c}$ & $10,61 a$ \\
\hline $\mathrm{AOC}$ & $45,21 d$ & $6,47 \mathrm{c}$ & $0,004 d$ & $0,036 \mathrm{c}$ & $0,123 b c$ & $0,008 \mathrm{~b}$ & $3,81 \mathrm{~d}$ & $27,67 \mathrm{c}$ & $6,98 \mathrm{~b}$ \\
\hline \multicolumn{10}{|c|}{ Fuente: Escobar (2011). } \\
\hline $\begin{array}{l}\text { Area Foliar (AF), } \\
\text { (TCR), Tasa de Cr } \\
\text { Foliar (LNAF), Pes }\end{array}$ & $\cos (\mathrm{F}$ & la & Area & Foliar E & (6) & $\pi$ & de Creci & . & $\begin{array}{l}\text { tivo } \\
\text { rea }\end{array}$ \\
\hline
\end{tabular}

En general, los mejores resultados de las variables de crecimiento en la etapa vegetativa se dieron en el tratamiento químico y mezcla 1, lo que indica que el abono orgánico tuvo un efecto igual al químico; así como en el maíz, la mezcla 1 mostró los valores más altos durante el crecimiento de los cultivos, como ya se mencionó este efecto puede deberse a la composición de la gallinaza. En la etapa de floración (Tabla 9) la mezcla 2 fue la que mejor respondió en las variables AF, PS, IAF, TCC, TAN, LNAF y AFE.

Tabla 9. Variables de crecimiento etapa de floración en frijol

\begin{tabular}{|c|c|c|c|c|c|c|c|c|c|}
\hline \multirow[t]{2}{*}{ TRATAMIENTOS } & \multicolumn{9}{|c|}{ VARIABLES DE CRECIMIENTO } \\
\hline & $\mathrm{AF}$ & PS & IAF & TCR & $\mathrm{TCC}$ & TAN & LNAF & $\mathrm{PF}$ & AFE \\
\hline FQ & $132,61 \mathrm{a}$ & $26,98 b$ & $0,015 a$ & $0,07 a$ & $0,59 b$ & $0,012 b$ & $5,66 a$ & $38,44 b c$ & $8,55 c$ \\
\hline $\mathrm{M} z 1$ & $133,7 \mathrm{a}$ & $25,93 b$ & $0,013 b$ & $0,08 \mathrm{a}$ & $0,57 b$ & $0,011 b$ & $4,80 b$ & $63,88 \mathrm{a}$ & $11,05 b$ \\
\hline $\mathrm{M} z 2$ & $128,4 a$ & $28,71 \mathrm{a}$ & $0,014 a$ & $0,056 \mathrm{~b}$ & $0,65 a$ & $0,014 a$ & $5,21 \mathrm{a}$ & $55,71 b$ & $14,54 \mathrm{a}$ \\
\hline $\mathrm{Mz3}$ & $95,66 \mathrm{~b}$ & $12,11 \mathrm{c}$ & $0,011 b$ & $0,048 b c$ & $0,321 \mathrm{c}$ & $0,013 a$ & $4,55 b$ & $41,11 b$ & $12,86 b$ \\
\hline SF & $60,31 c$ & $6,22 \mathrm{~d}$ & $0,010 b$ & $0,046 b c$ & $0,111 d$ & $0,051 \mathrm{c}$ & $3,95 \mathrm{c}$ & $37,63 \mathrm{c}$ & $8,12 \mathrm{c}$ \\
\hline $\mathrm{AOC}$ & $58,11 \mathrm{c}$ & $10,61 \mathrm{c}$ & $0,010 \mathrm{~b}$ & $0,039 c$ & $0,481 \mathrm{c}$ & $0,081 b c$ & $3,92 \mathrm{bc}$ & $39,81 \mathrm{~b}$ & $10,72 b c$ \\
\hline Fuente: Escobar (2011 & & & & & & & & & \\
\hline \multicolumn{10}{|c|}{ Letras diferentes indican diferencias significativas $p<0,05$. } \\
\hline $\begin{array}{l}\text { Área Foliar (AF), Pe } \\
\text { (TCR), Tasa de Cre } \\
\text { Foliar (LNAF), Peso }\end{array}$ & $\begin{array}{l}\text { so Seco (P } \\
\text { cimiento del } \\
\text { Fresco (PF) }\end{array}$ & $\begin{array}{l}\text { S) de la } \\
\text { Cultivo (1 } \\
\text { de la plan }\end{array}$ & $\begin{array}{l}\text { olanta, Ind } \\
\text { (CC). Tas } \\
\text { ta y Area }\end{array}$ & $\begin{array}{l}\text { dice de } A \\
\text { a de Asin } \\
\text { Foliar Es }\end{array}$ & $\begin{array}{l}\text { a Foliar } \\
\text { ación Ne } \\
\text { cífica (A }\end{array}$ & $\begin{array}{l}\text { F), Tasa } \\
\text { TAN), Lo }\end{array}$ & $\begin{array}{l}\text { le Crec } \\
\text { aritmo }\end{array}$ & $\begin{array}{l}\text { ento Rela } \\
\text { tural del } A\end{array}$ & $\begin{array}{l}\text { tivo } \\
\text { rea }\end{array}$ \\
\hline
\end{tabular}

La Tabla 10 presenta comparativamente el número promedio de vainas de fríjol por planta y peso promedio de vainas por planta para cada tratamiento. El número no presenta diferencias significativas $(p<0,005)$ entre tratamientos; con respecto al peso, la mezcla 2 obtuvo el mayor valor y presentó diferencias significativas $(p<0,005)$ con respecto al resto de tratamientos. El segundo valor lo obtuvieron las mezclas 1 y 3. 
Tabla 10. Variables de rendimiento del producto final en fríjol

\begin{tabular}{ccc}
\hline TRATAMIENTOS & No. de vainas & Peso vainas \\
\hline FQ & $17 \mathrm{a}$ & $46,8 \mathrm{bc}$ \\
Mz1 & $21,2 \mathrm{a}$ & $68,7 \mathrm{~b}$ \\
Mz2 & $24,2 \mathrm{a}$ & $113,8 \mathrm{a}$ \\
Mz3 & $18,2 \mathrm{a}$ & $69,5 \mathrm{~b}$ \\
SF & $14,5 \mathrm{a}$ & $21 \mathrm{c}$ \\
AOC & $31,2 \mathrm{a}$ & $39,2 \mathrm{bc}$ \\
\hline Fuente: Autores (2011). & &
\end{tabular}

Letras diferentes indican diferencias significativas $p<0,05$.

La mezcla 2 presentó el mejor resultado sobre el rendimiento del fríjol, esto puede asociarse al hecho de que el fríjol es una leguminosa y por lo tanto en la facilidad de colonización de algunas especies de bacterias en sus raíces, facilitando la mineralización de la materia orgánica y por tanto la disponibilidad de nutrimentos. Estrada y Peralta (2001) evaluaron el efecto de dos tipos de fertilizantes orgánicos (gallinaza y bovinaza) y un mineral en el crecimiento y rendimiento del cultivo de fríjol común (Phaseolus vulgaris L.) variedad DOR-364, postrera, obteniendo los mejores resultados con los fertilizantes orgánicos. Estos autores afirman que la presencia de sustratos orgánicos potencia el crecimiento de los microorganismos, los cuales inmovilizan los nutrimentos al incrementar su biomasa, los cuales más adelante pueden estar disponibles a otros microorganismos y a las plantas durante su ciclo de crecimiento.

Salas y Ramírez (2001), indican que los abonos orgánicos se pueden clasificar como superiores, iguales o inferiores respecto al tratamiento de fertilización química utilizado sobre el cultivo.

\section{CONCLUSIONES}

En este bioensayo se observó que el efecto de las mezclas en el cultivo de maíz y fríjol fue igual y superior al tratamiento del fertilizante químico. De esta forma, se demostró que los abonos orgánicos pueden igualar o superar a los fertilizantes químicos en el suplemento de nutrimentos a corto plazo.

El tratamiento que generó mayor rendimiento en el maíz fue la mezcla 3 , esto en parte porque su composición es de estiércoles (bovinaza y gallinaza), que son fertilizantes orgánicos relativamente concentrados y de rápida acción y sus nutrientes se encuentran como compuestos asimilables por la planta.

En el bioensayo se observó que el efecto de las mezclas 2 y 3 sobre el cultivo de maíz y fríjol fue similar al tratamiento del fertilizante químico, indicando que los abonos orgánicos son una alternativa viable y sostenible en condiciones de campo. 


\section{AGRADECIMIENTOS}

Los autores expresan agradecimientos al Centro de Investigaciones de la Universidad del Tolima, a la Red Alma Máter y a la Universidad de Cundinamarca, por las facilidades y financiamiento otorgado para los estudios básicos que dieron origen al presente artículo.

\section{REFERENCIAS}

- Bertsch, F. (2003). Abonos orgánicos. Manejo de la fracción orgánica y de los aspectos biológicos del suelo. Curso: Fertilizantes, características y manejo. Centro de Investigaciones Agronómicas. Universidad de Costa Rica. p. 112-130.

- Butler, T.J.; McFarland, M.L. y Muir, J.P. (2000). Using dairy manure compost for corn production. Texas water resources institute. p. 911.

- Cegarra, J. (1994). Fraccionamiento de fertilizantes orgánicos y de sus productos de humificación. Tesis doctoral. Universidad Autónoma de Madrid, España. p. 60-71.

- Díaz, R.M.; Acea, M.J. y Carballas, T. (1993). Microbiological characterization of four composted urban refuses. Biological wastes, 30(2), 89-100.

- Eghball, B.; Ginting, D. y Gilley, J.E. (2004). Residual Effects of Manure and Compost Applications on Corn Production and Soil Properties. Agron. J., 96, 442-447.

- Escobar, N. (2011). Caracterización de la población microbiana en el proceso de compostaje con sustratos provenientes de zonas cafeteras de Cundinamarca y Tolima. Tesis de Maestría en Ciencias Biológicas, Universidad del Tolima, Ibagué. 218 p.

- Estrada, M.E. y Peralta, J.R. (2001). Evaluación de dos tipos de fertilizantes orgánicos (gallinaza y estiércol vacuna) y un mineral en el crecimiento y rendimiento del cultivo de fríjol común (Phaseolus vulgaris L.) variedad DOR-364, postrera. Trabajo de grado. Facultad de Agronomía, Universidad Nacional Agraria, Nicaragua. p. 29-34.

- García, C. y Monje, N. (1995). Agricultura Orgánica. Costa Rica: EUNED. p. 457.

- Gómez, J. (2000). Abonos Orgánicos. Manual para productores. Cali. p. 107.

- Hunt, R. (2002). Plant growth analysis: individual plants. London: Academic Press. p. 579-588.

- Illner, P.; Otto, A.; Mair, J.; Malin, C. y Farbmacher, S. (2007). Chemical and Biochemical Parameters During Composting of Lawn Clippings with Special Regard to the Efficiency of a Compost Starter Kit. Compost Science \& Utilization, 15(1), 40-46.

- Jia, L; Xiu-hong, X; Hong-tao, L, y Ying, X. (2011). Effect of microbiological inocula on chemical and physical properties and microbial community of cow manure compost. Biomass and Bioenergy, 35(8), 3433-3439.

- Julca-Otiniano, A.; Solano-Arrue, W. y Crespo-Costa, R. (2008). Crecimiento de Coffea arábica variedad Caturra amarillo en almácigos con substratos orgánicos en Chanchamayo, selva central del Perú. p. 1-17. Disponible en: http://www.lamolina.edu.pe/proyectos/cafe/pdfs/coffea.pdf 
- Kolmans, E. y Vásquez, D. (1996). Estiércol y compost. Manual de Agroecología Ecológica, 3, 101-105.

- Labrador, J. (2001). La materia orgánica en los agrosistemas. 2 ed. Madrid: Ministerio de Agricultura, Pesca y Alimentación - Ediciones Mundi-Prensa. p. 152-180.

- Liang, C.; Das, K.C. y McClendon, R.W. (2003). The influence of temperature and moisture contents regimes on the aerobic microbial activity of a biosolids composting blend. Bioresour. Technol, 86, 131-137.

- Mazzarino, M.J.; Laos, F.; Satti, P.; Roselli, L.; Moyano, S.; Tognetti, C. y Labud, V. (2005). Aprovechamiento integral de residuos orgánicos en el NO de Patagonia. Grupo de Suelos del CRUB (Universidad Nacional Comahue), Bariloche. p. 52-61.

- Pérez, A.; Hermann, V.; Alabouvette, C. y Steinberg, C. (2006). Response of soil microbial communities to compost amendments. Soil Biol. Biochem, 38, 460-470.

- Pierre, F.; Rosell, M.; Quiroz, A. y Grabada, Y. (2009). Evaluación química y biológica de compost de pulpa de café en Caspito municipio Andrés Eloy Blanco, Estado Lara, Venezuela. Revista Bioagro, 21(2), 105-110.

- Ramírez, H. (2005). Producción sostenible de hortalizas. En: CursoTaller Introductorio Producción Sostenible de Hortalizas. Posgrado en Agronomía. Universidad Centro Occidental Lisandro Alvarado, Barquisimeto. Edo. Lara. p. 1-51.

- Raviv, M.; Medina, S.; Krasnovsky, A. y Ziadna, H. (2004). Organic matter and nitrogen conservation in manure compost for organic agriculture. Compost Sei. Util, 12(I), 6-10.

- Salas, E. y Ramírez, C. (2001). Bioensayo microbiano para estimar los nutrimentos disponibles en los abonos orgánicos. Agronomía Costarricense, 25(2), 11-23.

- Soto, M.G. (2003). Abonos orgánicos: definiciones y procesos. Abonos orgánicos: principios, aplicaciones e impactos en la agricultura. San José, Costa Rica: Ed. Meléndez. p. 20-49.

- Zamora, G.A. y Benavides, V.A. (2002). Evaluación del efecto de la fertilización mineral y orgánica (gallinaza) en el crecimiento y rendimiento del cultivo de maíz (Zea mays L.), variedad NB-S en la estación experimental "La compañía". Trabajo de grado. Facultad de Agronomía, Universidad Nacional Agraria. p. 45-51.

1. Profesora investigadora, Universidad de Cundinamarca. Profesorannaescobar@hotmail.com

2. Profesor Asociado, Grupo de Investigación Sistemas Agroforestales Pecuarios, Universidad del Tolima, Ibagué. jrmora@ut.edu.co

3. Profesor asistente, Departamento de Sanidad Animal, Universidad del Tolima, Ibagué.njromeroj@ut.edu.co

Para citar este artículo: Escobar, N., Mora, J. \& Romero, N. (2013). Respuesta agronómica de Zea mays L. y Phaseolus vulgaris L. a la fertilización con compost. Revista Luna Azul, 37, 1829. Recuperado de http://lunazul.ucaldas.edu.co/index.php?option=content\&task=view\&id=841 